BNL -45269

DE9 10011.33

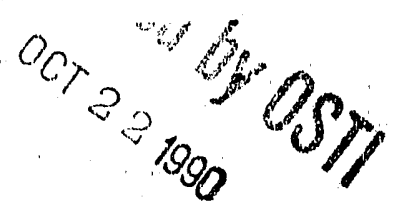

Intrabcam Scattering and the Beam Life-Time

J. Wei and A. G. Ruggiero

September 1990

R H I P P O J C T

Brookhaven National Laboratory

Associated Universities, Inc.

Upton, NY 11973

MASTER

Under Contract No. DE-AC02-76CH 00016 with the

UNITED STATES DEPARTMENT OF ENERGY

DISTRIBUTION OF THIS DOCUMENT IS UNLIMITI? 


\title{
Intrabeam Scattering and the Beam Life-Time
}

\author{
J. Wei and A.G. Ruggiero \\ Accelerator Development Department \\ Brookhaven National Laboratory \\ Upton, New York 11973
}

\begin{abstract}
The Fokker-Planck equation is derived to describe the time evolution of the longitudinal density distribution of a bunched hadron beam in the presence of intrabeam scattering. A computer program has been developed to numerically solve this equation. Both the beam loss and bunch-shape evolution are investigated for the ${ }^{197} \mathrm{Au}^{79+}$ beams during the 10-hour storage in the proposed RHIC collider.
\end{abstract}

\section{DISCLAIMER}

This report was prepared as sal account of work sponsored by an agency of the United States Government. Neither the United States Government nor any agency thereof, nor any of their employees, miakes any warranty, express or implied, or assumes any legal liability or responsibility for the accuracy, completeness, or usefulness of any information, apparatus, product, or process disclosed, or represents that its ase would not infringe privately owned rights. Reference herein to any specific commercial product, process, or service by trade name, trademark, manufacturer, or otherwise does not necessarily constitute or imply its endorsement, recommendation, or favoring by the United States Government or any agency thereof. The views and opinions of authors expressed hereit do not necessarily state or reflect those of the United States Government or any agency thereof. 


\section{Introduction}

The problem of intrabeam scattering, namely the Coulomb interaction between the particles in a beam, has been explored by many authors. Most of the theories ${ }^{1-4}$ developed on this subject are concerned with the growth of the rms beam dimensions under the assumption that the particle distribution remains Gaussian in both transverse and longitudinal phase space, often disregarding aperture limitations and particle loss. Previous studies $^{5}$ using this kind of theory indicate that during the 10-hour storage of the intense heavy-ion bunclıes in the RHIC, the growth of longitudinal bunch area and, consequently, the requirement on the rf voltage, are of primary concern. On the other hand, because the bunch area is in most cases comparable to the rf-bucket area, it is expected that particle loss through the edge of the rf bucket is appreciable. Under this circumstance, intrabeam-scattering calculation without taking into account the beam loss might not be adequate to describe the bunch behaviour.

This paper presents a new approacin to the problem based on the Fokker-Planck equation ${ }^{6-10}$ for the density distribution function of the particles of the bunched beam in the presence of intrabeam scattering. Section II introduces the simplified transport equation which, in terms of the action variable $J$, describes the time evolution of the longitudinal distribution function. Both the dynamic friction and the diffusion coefficients are obtained in terms of the distribution function itself. A computer program is develoned to solve the transport equation for given initial distribution and boundary conditions. Section III briefly addresses the numerical method used to evaluate the beam loss and to obtain the instantaneous particle distribution. Results are applied in section IV to the bunched beam of fully-stripped gold ions during the 10-hour storage in the RHIC collider. 


\section{Theoretical Approaches}

\section{A. The Fokker-Planck equation}

A well known approach to the problem of treating changes in a distribution function resulting from frequently occurring "events", each of which produces a small change in the configuration of the particles, is to use the Fokker-Planck equation. Let $x, x^{\prime} \equiv \frac{d x}{d z}$ and $y, y^{\prime}$ be the horizontal and vertical displacements and velocities, respectively, and $z$ the azirnuthal displacement along the closed orbit. The longitudinal motion of the particles can either be described by the rf phase deviation $\phi$ and the energy deviation $W=\Delta E / h \omega_{s}$ or, equivalently, the action-angle variables $Q$ and $J .{ }^{11}$ Here $h$ is the harmonic number, $\triangle E$ is the deviation from the syrichronous energy, and $\omega_{s}$ is the synchronous revolution frequency.

Longitudinal distribution function $\Psi_{T, L}\left(x, x^{\prime}, y, y^{\prime}, Q, J ; t\right)$ is defined as the number of particles per unit volume in the 6-dimensional phase space. The Fokker-Planck equation gives time rate of change of $\Psi_{T, L}$ due to intrabeam scattering in the laboratory frame as $\frac{\partial \Psi_{T, L}}{\partial t}=-\frac{\partial}{\partial x^{\mu}}\left(\Psi_{T, L} \frac{\left\langle\Delta x^{\mu}\right\rangle_{C}}{\Delta t}\right)+\frac{1}{2} \frac{\partial^{2}}{\partial x^{\mu} \partial x^{\nu}}\left(\Psi_{T, L} \frac{\left\langle\Delta x^{\mu} \Delta x^{\nu}\right\rangle_{C}}{\Delta t}\right), \quad \mu, \nu=x, x^{\prime}, y, y^{\prime}, Q, J$,

where the time interval $\Delta t$, as observed in the laboratory frame, is long compared with the correlation time of the intrabeam-scattering process but short compared with other time scales. $\Delta x^{\mu}$ is the increment of $x^{\mu}$ during $\Delta t .\langle\rangle_{C}$ indicates the average over collision events. The derivation of this equation is based on the justified assumption that the distant collisions are of predominant importance, which implies that small changes in $x^{\mu}$ are the most probable and that terms involving higher powers of $\Delta x^{\mu}$ contribute negligibly to $\frac{\partial \Psi_{T, L}}{\partial t}$.

Rewrite the distribution function as the product of the longitudinal and the normal- 
ized transverse distribution function,

$$
\Psi_{T, L}\left(x, x^{\prime}, y, y^{\prime}, Q, J ; t\right)=\rho_{H}\left(x, x^{\prime} ; t\right) \rho_{V}\left(y, y^{\prime} ; t\right) \Psi_{L}(Q, J ; t)
$$

where $H$ and $V$ refer to the horizontal and vertical dimensions, respectively. The 6dimensional Fokker-Planck equation can be reduced to a 2-dimensional equation by integrating both sides of eq. 1 over all the transverse variables $x, x^{\prime}, y$, and $y^{\prime}$,

$$
\begin{array}{r}
\frac{\partial \Psi_{L}}{\partial t}=-\frac{\partial}{\partial x^{\mu}}\left(\Psi_{L} \frac{\left\langle\Delta x^{\mu}\right\rangle_{C, T}}{\Delta t}\right)+\frac{1}{2} \frac{\partial^{2}}{\partial x^{\mu} \partial x^{\nu}}\left(\Psi_{L} \frac{\left\langle\Delta x^{\mu} \Delta x^{\nu}\right\rangle_{C, T}}{\Delta t}\right) \\
\mu, \nu=Q, J
\end{array}
$$

where the subscript $T$ denotes the average over all the transverse variables.

The time for int abeam scattering to produce appreciable effect (or the time of relaxation) is typical $y$ much lorger than the synchrotron-oscillation period, which is again much longer than the correlation time of the collision process. In this case, eq. 3 can be further simplified by averaging over the angle variable $Q$ for one synchrntron-oscillation period:

$$
\frac{\partial \Psi(J ; t)}{\partial t}=-\frac{\partial}{\partial J}\left(\Psi(J ; t) \frac{\langle\Delta J\rangle_{C, T, Q}}{\Delta T}\right)+\frac{1}{2} \frac{\partial^{2}}{\partial J^{2}}\left(\Psi(J ; t) \frac{\left\langle(\Delta J)^{2}\right\rangle_{C, T, Q}}{\Delta T}\right)
$$

where the subscript $Q$ denotes an additional average over $Q$, and $\Delta T$ corresponds to a time long compared with the synchrotron-oscillation period, but still short compared with the relaxation time. $\Psi(J ; t)$ is the averaged distribution function in longitudinal phase space expressed in term $\mathrm{s}$ of the phase-space area $J$ enclosed by the particle performing synchrotron oscillation.

For the examination of eq. 4 , the coefficients must be expressed in approachable forms. Consider the Coulomb interaction of a "test" particle with the "media" particles of the beam. Using the action-angle variables, the longitudinal equations of motion of this test 
particle can in general be described by

$$
\left\{\begin{array}{l}
\dot{Q}=\frac{\Omega_{s}(J)}{2 \pi}+\frac{\Delta Q}{\Delta t} \\
\dot{J}=\frac{\Delta J}{\Delta t}
\end{array}\right.
$$

where the synchrotron-oscillation frequency $\Omega_{s}$ is a function of $J$ orly. For simplicity, the angle variable $Q$ is normalized to 1 so that 7 presents the phase-space area.

In eq. $5, \frac{\Delta Q}{\Delta t}$ and $\frac{\Delta J}{\Delta t}$ are the time rates of change in $Q$ and $J$ due to the collisions. Since the change in distribution is small within one synchrotron-oscillation period, both $\frac{\Delta Q}{\Delta t}$ and $\frac{\Delta J}{\Delta t}$ can be written as periodic functions in $Q$. It is thus straightforward to verify that the average of these quantities can be expressed as

$$
\frac{\langle\Delta J\rangle_{C, T, Q}}{\Delta T} \approx F^{0}(J ; t)+F^{1}(J ; t)
$$

where

$$
F^{0}(J ; t)=\left.\int_{0}^{1} d Q \frac{\langle\Delta J\rangle_{C, T}}{\Delta t}\right|_{0}, \quad F^{1}(J ; t)=\frac{1}{2} \frac{d}{d J}\left[\left.\int_{0}^{1} d Q \frac{\left\langle(\Delta J)^{2}\right\rangle_{C, T}}{\Delta t}\right|_{0}\right]=\frac{1}{2} \frac{d D(J ; t)}{d J},
$$

and

$$
\frac{\left\langle(\Delta J)^{2}\right\rangle_{C, T, Q}}{\Delta T} \approx D(J ; t)=\left.\int_{0}^{1} d Q \frac{\left\langle(\Delta J)^{2}\right\rangle_{C, T}}{\Delta t}\right|_{0} .
$$

The subscript 0 in eqs. 7 and 8 implies that the integration ove: $Q$ is performed along the contours of particle motion in the absence of intrabeam scattering for one synchrotronoscillation period.

As a result of these considerations, the Fokker-Planck equation can finally be simplified as a partial differential equation in $J$ and $t$ with simple coefficients for the determination of the distribution function $\Psi$,

$$
\frac{\partial \Psi}{\partial t}=-\frac{\partial}{\partial J}\left(F^{0} \Psi\right)+\frac{1}{2} \frac{\partial}{\partial J}\left(D \frac{\partial \Psi}{\partial J}\right) \text {. }
$$


The boundary condition to this equation is that $\Psi$ vanishes at the separatrix $\hat{J}$, and that the flux vanishes at $J=0$, i.e.

$$
\left\{\begin{array}{l}
J=0: \quad-F^{0} \Psi+\frac{D}{2} \frac{\partial \Psi}{\partial J}=0 \\
J=\hat{J}: \quad \Psi=0 .
\end{array}\right.
$$

The coefficient $D$ of diffusion is alway positive, indicating the tendency of bunch-area growth due to intrabeam scattering. The existence of a non-zero coefficient $F^{0}$ of dynamic friction reflects the non-uniform environment seen by the test particles in velocity space. Both $F^{0}$ and $D$ are dependent on the particle distribution itself. Their explicit expressions will be derived in the following subsections.

\section{B. The canonical transformation}

The increment $\Delta J$ in the action $J$ of the test particle can be related to that in the energy deviation $W$ by a transformation. In terms of $\phi$ and $W$, the longitudinal equations of motion become

$$
\left\{\begin{aligned}
\dot{W} & =\frac{q e \hat{V}}{2 \pi h}\left[\sin \left(\phi_{s}+\phi\right)-\sin \phi_{s}\right]+\frac{\Delta W}{\Delta t} \\
\dot{\phi} & =\frac{h^{2} \omega_{s}^{2} \eta}{E \beta^{2}} W
\end{aligned}\right.
$$

where $\hat{V}$ is the peak voltage, $\eta=1 / \gamma_{T}^{2}-1 / \gamma^{2}, \gamma_{T}$ is the transition energy, $E=A m_{0} c^{2} \gamma$ and $\beta c$ are the synchronous energy and velocity, respectively, and $q$ and $A$ are the charge and atomic number of the particles. For simplicity, the synchronous phase $\phi_{\mathrm{s}}$ will be taken as $\pi$, which represents the storage mode above transition in the RHIC.

Regarding the rate of energy increment $\frac{\Delta W}{\Delta t}$ due to intrabearn scattering as a perturbation, the unf srturbed particle motion is derivable from an Hamiltonian

$$
\dot{H}(\phi, W)=C_{W} W^{2}+C_{\phi} \sin ^{2} \frac{\phi}{2}
$$


where

$$
C_{W}=\frac{h^{2} \omega_{s}^{2} \eta}{2 E \beta^{2}} \quad \text { and } \quad C_{\phi}=\frac{q e \hat{V}}{\pi h}
$$

may be time dependent. The transformation to the action-angle variables may be achieved by means of a generating function of Goldstein's second type, ${ }^{12}$

$$
F_{2}(\phi, J ; t)=\int^{\phi} \sqrt{\frac{1}{2 \pi C_{W}} \int^{J} \Omega_{s}\left(J^{\prime}\right) d J^{\prime}-\frac{C_{\phi}}{C_{W}} \sin ^{2} \frac{\phi^{\prime}}{2}} d \phi^{\prime} .
$$

Obviously, the action variable $J$ is an invariant of motion in the absence of intrabeam scattering,

$$
J=\oint W d \phi=8 \sqrt{\frac{C_{\phi}}{C_{W}}}\left[\left(k^{2}-1\right) \mathrm{K}(k)+\mathrm{E}(k)\right], \quad k=\sqrt{\frac{H}{C_{\phi}}} \leq 1,
$$

where

$$
\mathrm{K}(k)=\int_{0}^{\frac{\pi}{2}} \frac{d t}{\sqrt{1-k^{2} \sin ^{2} t}} \text {, and } \mathrm{E}(k)=\int_{0}^{\frac{\pi}{2}} \sqrt{1-k^{2} \sin ^{2} t} d t
$$

are the complete elliptical integrals ${ }^{13}$ of first and second kind. The synchrotron-oscillation frequency

$$
\Omega_{s}=2 \pi \dot{Q}=\frac{\pi \sqrt{C_{W} C_{\phi}}}{2 \mathrm{~K}(k)}, \quad k \leq 1,
$$

is a monotonic function of $J$. At the boundary (separatrix) of the stable region, $\hat{J}=$ $8 \sqrt{\frac{C_{\phi}}{C_{W}}}$, and $\Omega_{s}(\hat{J})=0$.

The contribution $\frac{\Delta W}{\Delta t}$ from intrabeam. scattering is a function of $\phi$ and $W$ of the test particle. This part is not derivable from an Hamilconian. Due to this contribution, the action $J$ generally increases with time, which results a growth in bunch area. Using eq. 14 and the canonical relations, the rate of increment in $J$ can be expressed in terms of $\frac{\Delta W}{\Delta t}$ as

$$
\frac{\Delta J}{\Delta t}=\left.\frac{\partial W}{\partial J}\right|_{\phi} ^{-1} \frac{\Delta W}{\Delta t} .
$$

It follows from ref. 11 that the coefficient in eq. 17 can be written as a series expansion using the order parameter

$$
\xi=\exp \left[-\pi \mathrm{K}^{\prime}(k) / \mathrm{K}(k)\right], \quad \mathrm{K}^{\prime}(k)=\mathrm{K}\left(\sqrt{1-k^{2}}\right)
$$


as

$$
\left.\frac{\partial W}{\partial J}\right|_{\phi} ^{-1}=8 k \mathrm{~K}(k) \cos 2 \pi Q\left[1-4 \xi \sin ^{2} 2 \pi Q+O\left(\xi^{2}\right)\right],
$$

where $k$ is a function of $J$ only (eq. 14). Note that $\xi$ becomes significant only near the separatrix (fig. 1 in ref.11).

\section{Rutherfold scattering in the rest frame}

It is convenient to evaluate the rate of energy increment $\frac{\Delta W}{\Delta t}$ in the rest frame of the synchronous particle where the motion of particles in the bunch is non-relativistic. The four components of the momentum vector in the rest frame can be expressed in terms of those in the laboratory frame by means of the Lorentz transformation,

$$
\left\{\begin{aligned}
\bar{p}_{x, y} & =p_{x i, y} \\
\bar{p}_{z} & \approx \frac{A m_{0} c}{\beta} \frac{\Delta \gamma_{z}}{\gamma}, \\
\bar{E} & \approx A m_{0} c^{2}
\end{aligned}\right.
$$

where the bar indicates the value in the rest frame. $\beta$ and $\gamma$ again represent the synchronous values.

Let $\mathbf{u}=\overrightarrow{\mathbf{v}}_{1}-\overrightarrow{\mathbf{v}}_{2}$ denote the relative velocity between the test particle 1 and a media particle 2 in the beam observed in the rest frame. $u=|\mathbf{u}| \mathrm{i}$ " related to the three momentum components in the laboratory frame by

$$
u \approx \beta c \sqrt{\left(\frac{\Delta p_{1}}{p}-\frac{\Delta p_{2}}{p}\right)^{2}+\gamma^{2}\left(x_{1}^{\prime}-x_{2}^{\prime}\right)^{2}+\gamma^{2}\left(y_{1}^{\prime}-y_{2}^{\prime}\right)^{2}}
$$

To evaluate $\frac{\Delta W}{\Delta t}$, Rutherford's formula is used for the cross section of the Coulomb scattering,

$$
\sigma(u, \theta)=\frac{q^{4} e^{4}}{A^{2} m_{0}^{2} u^{4} \sin ^{4} \theta / 2}
$$

where $\theta$ is the angle through which the velocity vector $u$ undergoes a rotation during the collision. Integrating over both $\theta$ and the azimuthal scattering angle, the change in 
the longitudinal component of the velocity of the test particle and its square per unit rest-frame time can be showri as

$$
\left\langle\Delta \ddot{v}_{z 1}\right\rangle_{\Omega}=-2 \Gamma \frac{u_{z}}{u^{3}}, \quad \text { and } \quad\left\langle\left(\Delta \ddot{v}_{z}\right)_{1}^{2}\right\rangle_{\Omega}=\Gamma^{4} \frac{u_{x}^{2}+u_{y}^{2}}{u^{3}},
$$

where

$$
\Gamma \equiv \frac{4 \pi q^{4} c^{4} \log }{A^{2} m_{0}^{2}}, \quad \log \equiv-\ln \sin \frac{\theta_{\min }}{2},
$$

and $\theta_{\min }$ is the minimum scattering angle. The Coulomb logarithm Log can be verified to be much larger than 1. This fact implies that the Fokker-Planck equation is a good approximation to describe the particle motion. Because of the insensitivity of Log to the precise value of $u$, a fixed value of 20 is currently designated to Log to simplify further development.

Based on the results of eq. 22 , it is possible to evaluate the time rate of average energy increment of the test particle in the laboratory frame by integrating over all the velocity components of the media particles involved in the collision $\left(x_{2}=x_{1}\right.$ and $\left.y_{2}=y_{1}\right)$,

$$
\begin{aligned}
\frac{\langle\Delta W\rangle_{C}}{\Delta t}= & \frac{1}{\gamma} \frac{\beta E}{h \omega_{s} c} \int d x_{\beta 2}^{\prime} \rho_{x_{\beta} x_{\beta}^{\prime}}\left(x_{\beta 2}, x_{\beta 2}^{\prime} ; t\right) \int d y_{2}^{\prime} \rho_{y y^{\prime}}\left(y_{2}, y_{2}^{\prime} ; t\right) \\
\frac{\frac{h}{\gamma R} \int d W_{2} \Psi\left[J\left(\phi_{1}, W_{2}\right)\right]\left\langle\Delta \bar{v}_{z 1}\right\rangle_{\Omega},}{\Delta t}= & \frac{1}{\gamma}\left(\frac{\beta E}{h \omega_{s} c}\right)^{2} \int d x_{2}^{\prime} \rho_{x_{\beta} x_{\beta}^{\prime}}\left(x_{\beta 2}, c_{\beta 2}^{\prime} ; t\right) \int d y_{2}^{\prime} \rho_{y y^{\prime}}\left(y_{2}, y_{2}^{\prime} ; t\right) \\
& \frac{h}{\gamma R} \int d W_{2} \Psi\left[J\left(\phi_{1}, W_{2}\right)\right]\left\langle\left(\Delta \bar{v}_{z}\right)_{1}^{2}\right\rangle_{\Omega} .
\end{aligned}
$$

Note that the horizontal displacement of the particle from the closed orbit is the sum of the contribution of betatron oscillation and momentum deviation,

$$
x=x_{,}+x_{p} \frac{\Delta p}{p}, \quad \text { and } \quad x^{\prime}=x_{\beta}^{\prime}+x_{p}^{\prime} \frac{\Delta p}{p},
$$

where $x_{p}$ is the horizontal dispersion function. The three integrals in eq. 23 refer to the horizontal, vertical, and longitudinal components of velocity of the media particles, 
respectively, and $2 \pi R$ is the circumference of the machine. Obviously, the evaluation of the first two integrals requires the knowledge of the transverse distribution which is generally time dependent.

\section{Evaluation of the dynamic-friction and the diffusion coefficients}

According to the previous studies ${ }^{5}$ on intrabeam scattering, the transverse emittance of the beam is expected to be much smaller than the transverse admittance during the entire 10-hour period of operation in the RHIC. Beam loss in transverse direction due to intrabeam scattering is therefore negligible. Under this circumstance, the distribution in horizontal and vertical phase space may be assumed as

$$
\begin{aligned}
& \rho_{x_{\beta} x_{\beta}^{\prime}}\left(x_{\beta}, x_{\beta}^{\prime} ; t\right)=\frac{\sqrt{1+\alpha_{x}^{2}}}{2 \pi \sigma_{x_{\beta}} \sigma_{x_{\beta}^{\prime}}} \exp \left[-\frac{1+\alpha_{x}^{2}}{2}\left(\frac{x_{\beta}^{2}}{\sigma_{x_{\beta}}^{2}}+\frac{2 \alpha_{x} x_{\beta} x_{\beta}^{\prime}}{\left.\left.\sqrt{1+\alpha_{x}^{2} \sigma_{x_{\beta}} \sigma_{x_{\beta}^{\prime}}}+\frac{x_{\beta}^{\prime 2}}{\sigma_{x_{\beta}^{\prime 2}}}\right)\right]}\right.\right. \\
& \rho_{y y^{\prime}}\left(y, y^{\prime} ; t\right)=\frac{\sqrt{1+\alpha_{y}^{2}}}{2 \pi \sigma_{y} \sigma_{y^{\prime}}} \exp \left[-\frac{1+\alpha_{y}^{2}}{2}\left(\frac{y^{2}}{\sigma_{y}^{2}}+\frac{2 \alpha_{y} y y^{\prime}}{\left.\left.\sqrt{1+\alpha_{y}^{2} \sigma_{y}^{\prime} \sigma_{y^{\prime}}}+\frac{y^{\prime 2}}{\sigma_{y^{\prime}}^{2}}\right)\right] .}\right.\right.
\end{aligned}
$$

The time dependence of the transverse distribution can be expressed through the normalized transverse emittance $\epsilon_{N x, y}$,

$$
\sigma_{x_{\beta}, y}=\sqrt{\frac{\beta_{x, y} \epsilon_{N x, y}(t)}{6 \beta \gamma}}, \quad \sigma_{x_{\beta}^{\prime}, y^{\prime}}=\sqrt{\frac{\left(1+\alpha_{x, y}^{2}\right) \epsilon_{N x, y}(t)}{6 \beta \gamma \beta_{x, y}}}
$$

where $\beta_{x, y}$ and $\alpha_{x, y}$ are the Courant-Snyder lattice parameters. The time variation in $\epsilon_{N x, y}$ is in principle determined by the instantaneous bunch configuration under intrabeam scattering.

Based on the above assumptions on the transverse distribution, the evolution of the longitudinal distribution function can be determined by the transport equation (eq. 9) under the boundary condition (eq. 10). Define dimensionless quantities

$$
\kappa=\frac{1}{\gamma}\left(\frac{\Delta p_{1}}{p}-\frac{\Delta p_{2}}{p}\right), \quad a=\frac{1}{2} \sqrt{\frac{6 \beta \gamma \beta_{x, y}}{\epsilon_{N x, y}}}, \quad b=\left|\frac{\gamma \kappa}{2 \sigma_{x_{\beta}}}\left(\beta_{x} x_{p}^{\prime}+\alpha_{x} x_{p}\right)\right| .
$$


Substituting eqs. 17 and 23 into eqs. 7 and 8 and employing the above definition, the coefficients $F^{0}$ and $D$ can finally be obtained by integrating over all the transverse components $\left(x_{\beta 1}, x_{\beta 1}^{\prime}, y_{1}\right.$, and $\left.y_{1}^{\prime}\right)$ of the test particle,

$$
F^{0}(J)=\left.\left.\int \frac{2 d z}{\pi R} \int_{0}^{\frac{1}{4}} d Q \frac{\partial W}{\partial J}\right|_{\phi} ^{-1}(Q, J) \int_{J_{\min }}^{j} \frac{\partial W}{\partial J}\right|_{\phi}\left(Q^{\prime}, J^{\prime}\right)\left[A_{F}\left(\kappa_{1}\right)+A_{F}\left(\kappa_{2}\right)\right] \Psi\left(J^{\prime}\right) d J^{\prime}
$$

and

$$
D(J)=\left.\int \frac{2 d z}{\pi R} \int_{0}^{\frac{1}{4}} d Q\left[\left.\frac{\partial W}{\partial J}\right|_{\phi} ^{-1}(Q, J)\right]^{2} \int_{J_{\min }}^{j} \frac{\partial W}{\partial J}\right|_{\phi}\left(Q^{\prime}, J^{\prime}\right)\left[A_{D}\left(\kappa_{1}\right)+A_{D}\left(\kappa_{2}\right)\right] \Psi\left(J^{\prime}\right) d J^{\prime}
$$

where $\kappa_{1,2}=\frac{h \omega_{s}}{\gamma \beta^{2} E}\left(W \mp W^{\prime}\right)$, and

$$
\begin{aligned}
& A_{F}(\kappa)=-2 \Gamma \frac{1}{\gamma} \frac{\beta E}{h \omega_{s} c} \frac{h}{\gamma R} \frac{1}{(\beta c \gamma)^{2}} \frac{1}{4 \pi \sigma_{x_{\beta}} \sigma_{y}} I_{F}(\kappa), \\
& A_{D}(\kappa)=\Gamma^{\prime} \frac{1}{\gamma}\left(\frac{\beta E}{h \omega_{s} c}\right)^{2} \frac{h}{\gamma R} \frac{1}{\beta c \gamma} \frac{1}{4 \pi \sigma_{x_{\beta}} \sigma_{y}} I_{D}(\kappa) .
\end{aligned}
$$

The first integral in eqs. 28 and 29 represents the average over the machine lattice; the second integral represents the average over synchrotron-oscillation period; while the third integral describes the contribution from particles of different action $J^{\prime}$ involved in the collision. Fig. 1 shows that for given $J$ and $Q$, the integration over $J^{\prime}$ is performed such that $k\left(J^{\prime}\right) \sin 2 \pi Q^{\prime} \approx \sin [\phi(Q, J) / 2]$, extending from $J_{m i n}$ to the separatrix $\hat{J}$, with $k\left(J_{\min }\right) \approx[\sin \phi(Q, J) / 2]$. It is evident that the time dependence of $F^{0}$ and $D$ is governed by the instantaneous longitudinal distribution function $\Psi$ and the transverse emittance.

For a round beam with $\beta_{x} / \epsilon_{N x} \sim \beta_{y} / \epsilon_{N y}$ on the average, the quantities $I_{F}$ and $I_{D}$ in eq. 30 can be obtained in integral forms,

$$
\begin{aligned}
& I_{F}(\kappa)=\frac{4 a^{2} \chi \lambda}{\pi} \int_{0}^{\infty} \frac{e^{-\rho^{2}} \mathrm{E}(r) \rho d \rho}{\left[(\rho-b)^{2}+\lambda^{2}\right]\left[(\rho+b)^{2}+\lambda^{2}\right]^{\frac{1}{2}}} \\
& I_{D}(\kappa)=\frac{4 a \chi}{\pi} \cdot \int_{0}^{\infty} \frac{e^{-\rho^{2}} \rho d \rho}{\left[(\rho+b)^{2}+\lambda^{2}\right]^{\frac{1}{2}}}\left[\mathrm{~K}(r)-\frac{\lambda^{2} \mathrm{E}(r)}{(\rho-b)^{2}+\lambda^{2}}\right]
\end{aligned}
$$




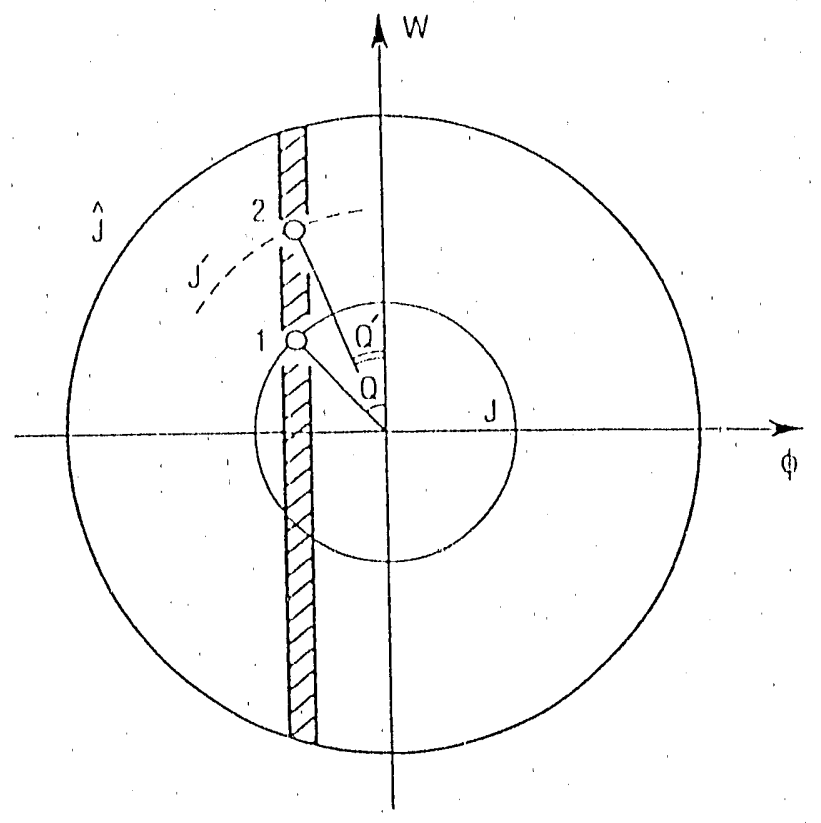

Figure 1: Integration in longitudinal phase space.

where

$$
\lambda=a \kappa, \quad r=\left[\frac{4 b \rho}{(\rho-b)^{2}+\lambda^{2}}\right]^{\frac{1}{2}}, \text { and } \chi=\exp \left[-\left(\frac{x_{p} \gamma \kappa}{2 \sigma_{x_{\beta}}}\right)^{2}\right] .
$$

If, as in many cases, $\beta_{x} x_{p}^{\prime}+\alpha_{x} x_{p} \cdot \cdot 0$, the integrals in eq. 32 can be performed to yield simpler expressions in terms of the error function $\Phi$,

$$
\begin{aligned}
& I_{F}(\kappa)=2 a^{2} \operatorname{sgn}(\kappa) \chi\left\{1-\sqrt{\pi}|\lambda| e^{\lambda^{2}}[1-\Phi(\lambda)]\right\} \\
& I_{D}(\kappa)=a \chi\left\{\sqrt{\pi}\left(1+2 \lambda^{2}\right) e^{\lambda^{2}}[1-\Phi(\lambda)]-2|\lambda|\right\}
\end{aligned}
$$

where $\operatorname{sgn}(\kappa)$ is 1 if $\kappa \geq 0$, and is -1 if otherwise. $\chi$ represents the damping effect of the dispersion $x_{p}$ on the diffusion process. Intuitively, the dispersion effectively increases the transverse beam size $\sigma_{x_{\beta}}$ by the amount $x_{p} \sigma_{\Delta p / p}$. The diffusion rate is therefore decreased accordingly.

Analytical solutions to the non-linear partial differential equation (eq. 9 with eqs. 28 , 29,30 and 33 ) with the boundary condition (eq. 10) and the initial condition $\Psi(J ; 0)$, are in general difficult to obtain. Fortunately, computer algorithm can be developed to 
numerically achieve an iterative solution $\Psi(J, t)$. Once $\Psi(J, t)$ is known, both beam loss and particle distribution in longitudinal space can be readily obtained.

\section{Computer Techniques}

The computer program originally developed for the investigation of the bunchedbeam stochastic, cooling ${ }^{11}$ has been modified to study the intrabeam scattering. First, the $J$-space $(0, \hat{J})$ is equally divided into $N_{J}$ bins of width $\Delta J$. Assumed as a truncated Gaussian in phase space, an initial distribution $\Psi\left(J_{i}, 0\right), i=1, \cdots, N_{J}$, is then generated. The evolution of the distribution function $\Psi(J ; t)$ is obtained by numerically iterating the transport equation which is written in a difference form, while keeping the boundary condition satisfied. The change in bunch area, i.e. the first moment $\left(\sum_{i=1}^{N_{J}} J_{i} \Psi\left(J_{i}\right) \Delta J\right)$ in $J$, is used to obtain the growth rate, while the zeroth moment $\left(\sum_{i=1}^{N_{J}} \Psi\left(J_{i}\right) \Delta J\right)$ in $J$ is used to determine the particle loss.

During the entire period the distribution in transverse phase space is assumed to be Gaussian. The instantaneous rate of change of the transverse emittance is provided by the standard intrabeam-scattering calculations. ${ }^{4}$

\section{Application to the RHIC}

Once the transport equation and the computer techniques are developed, the problem of beam life-time and bunch-area growth originated from intrabeam scattering can be readily approached. In this section, we apply the results to the storage of heavy-ion beams in the RHIC collider.

The heavy-ion beams will be stored in the RHIC for experiments at the energy of $100 \mathrm{GeV}$ per nucleon using the $160 \mathrm{MHz}, h=2052 \mathrm{rf}$ system. Consider the beam of one of the highest charge-state ions ${ }^{197} \mathrm{Au}^{79+}$ in the RHIC where intrabeam scattering is 
expected to be the severest. Each of the 57 or 114 bunches circulating in the ring contains $10^{9}$ particles. With the currently designed lattice, it is assumed that $\overline{\beta_{x, y}} \approx 25 \mathrm{~m}$, and that $x_{p} / \sqrt{\beta_{x}} \approx 0.22 \mathrm{~m}^{1 / 2}$. The initial bunch area is assumed to be $0.3 \mathrm{eV} \cdot \mathrm{s}$ per nucleon.

In the following, we study several operational scenarios for the RHIC storage using either a constant rf voltage, or a voltage programmed to achieve the so-called "tight bucket", with or without the initial blow-up of the transverse emittance.

\section{A. Constant voltage with initial emittance blow-up}

For the purpose of minimizing beam growth and luminosity variation during the storage, it is suggested that the transverse emittance should be initially blown-up to a normalized value of $60 \pi \mathrm{mm}$-mrad. Thereafter the growth in transverse emittance is small. ${ }^{14}$

Keeping the peak rf voltage at a constant value of $4.5 \mathrm{MV}$, fig. 2 shows the time evolution of the distribution function $\Psi_{0}(J)$ in $J$ during the 10-hour period of operation. The area of the $\mathrm{rf}$ bucket is about $1.5 \mathrm{eV} \cdot \mathrm{s} / \mathrm{amu}$. The initial time $t=0$ corresponds to the moment that the ion bunches are transferred from the $h=342 \mathrm{rf}$ system for acceleration to the $h=2052 \mathrm{rf}$ system for storage. Fig. 3 shows the initial and final line-density distribution as a function of the azimuthal displacement $(\phi)$ along the ring. It is indicated in fig. 4 that beam loss becomes significant after about 3 hours when the bunch area is comparable with the bucket area and, at the same time, when the growth in bunch length $\sigma_{l}$ starts to saturate (fig. 5). The total beam loss during the 10-hour period is about $20 \%$.

It is observed that the final distribution in longitudinal phase space $(\phi, W)$ is Gaussianlike, independent of the initial distribution. The final ratio of the bucket area to the average phase-space area $\langle J\rangle$ of the beam is about $3 \sim 4$, which again depends weakly on 


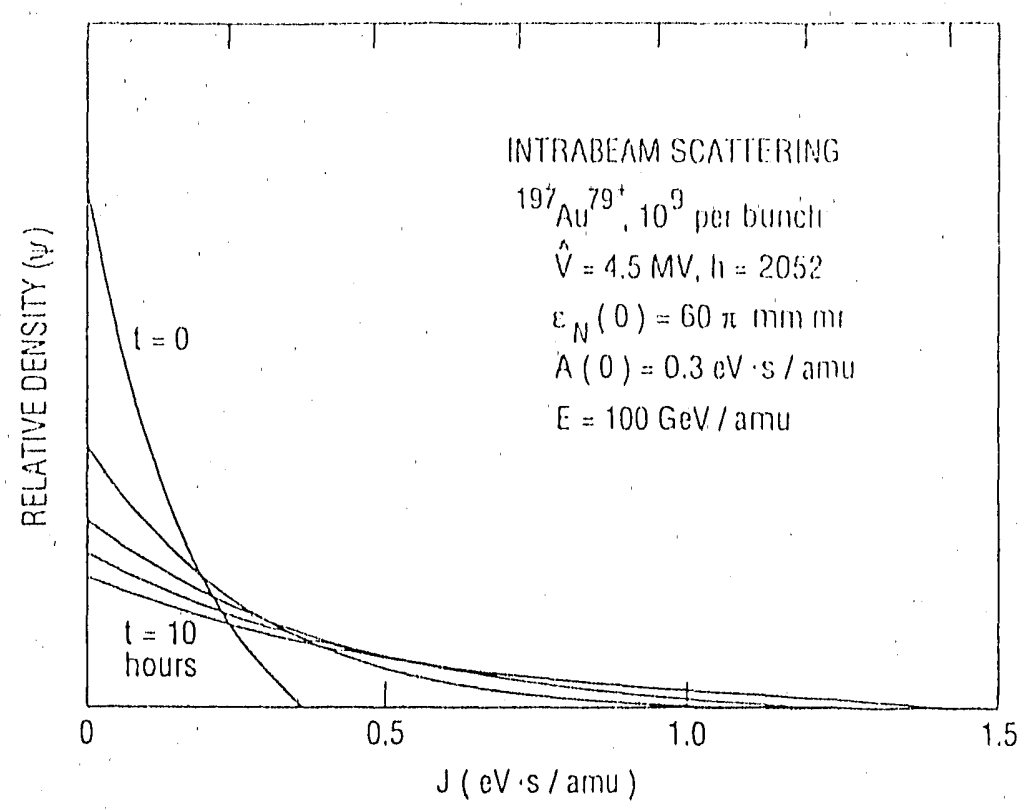

Figure 2: Evolution of the longitudinal distribution function $\Psi$ as a function of longitudinal phase space area $J$. $(t=0,2.5,5,7.5$, and 10 hour $)$.

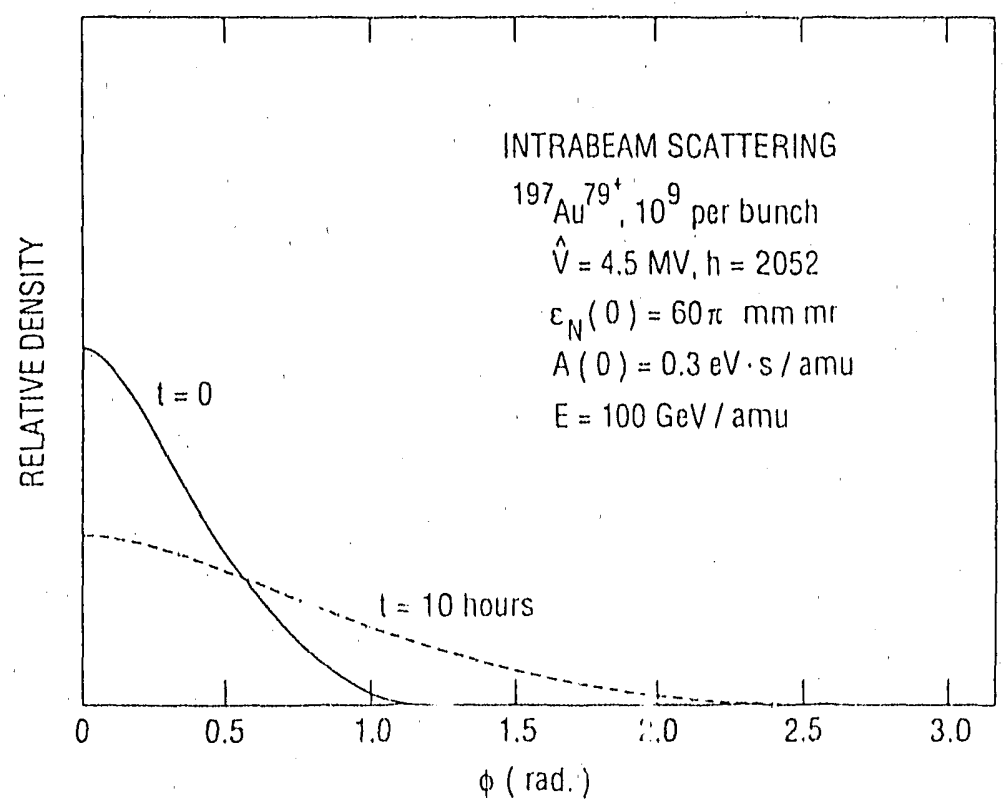

Figure 3: The initial and final density distribution along the azimuthal displacement $\phi$. 


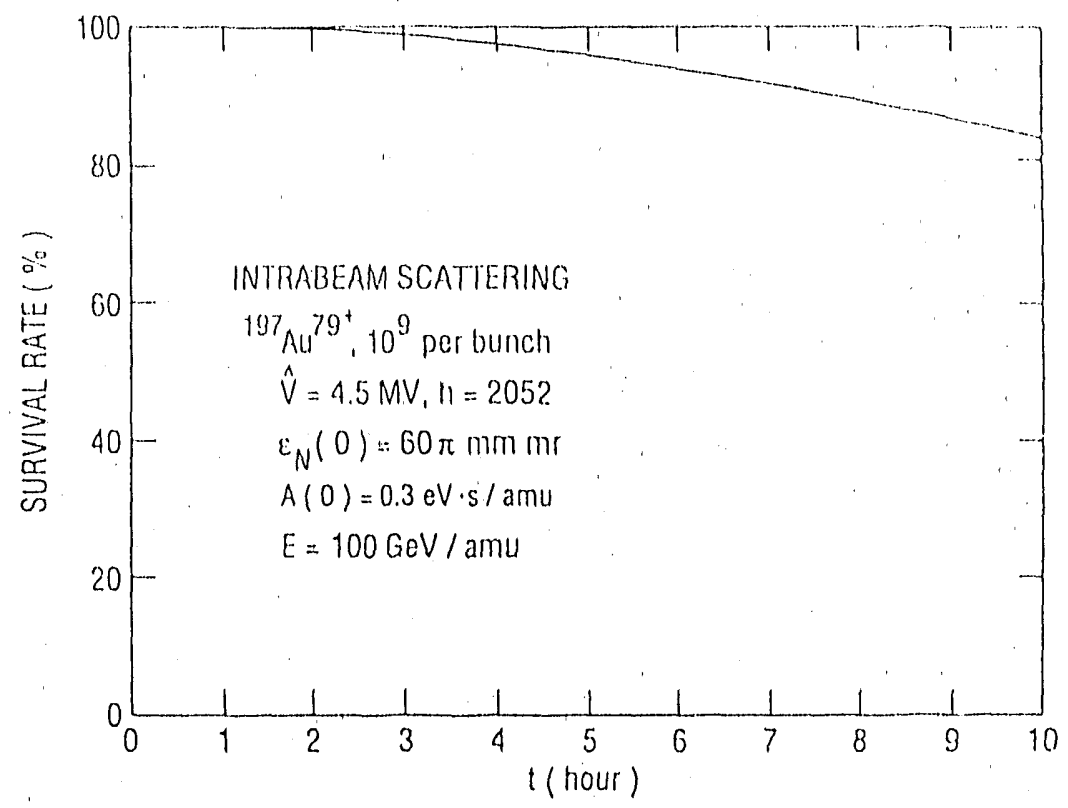

Figure 4: The beam urvival rate as a function of time during the 10-hour operation.

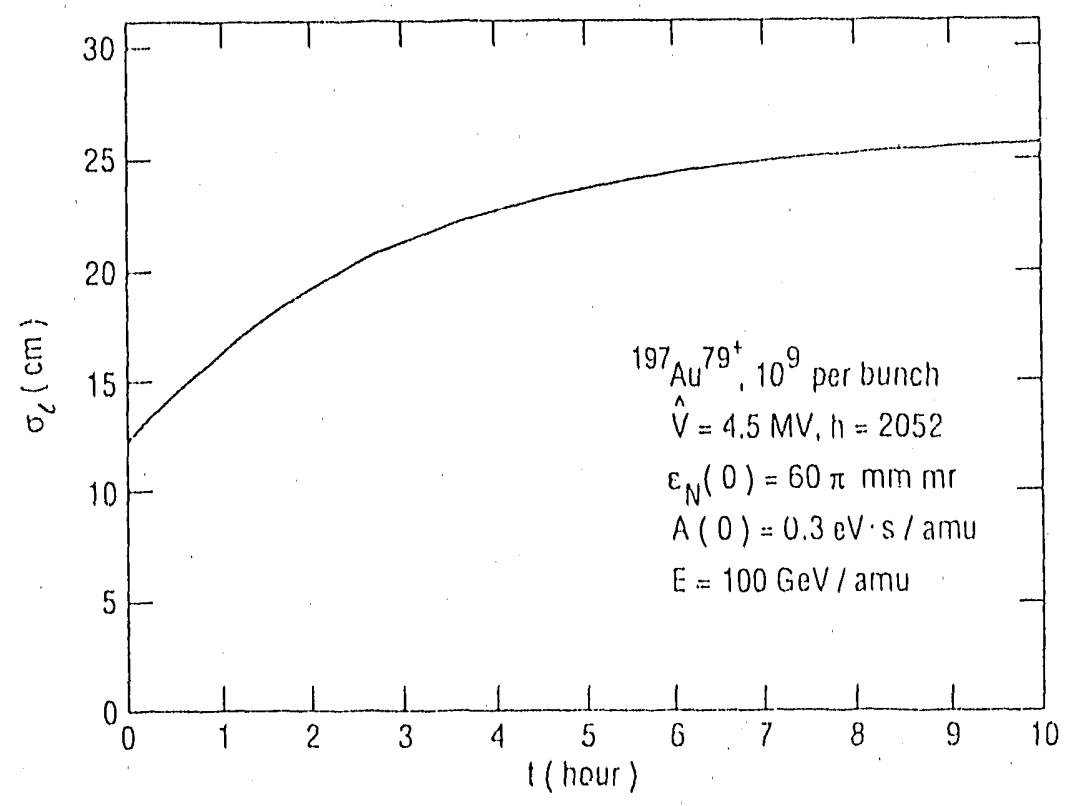

Figure 5: The growth in rms bunch length due to intrabeam scattering during the 10-hour operation. 


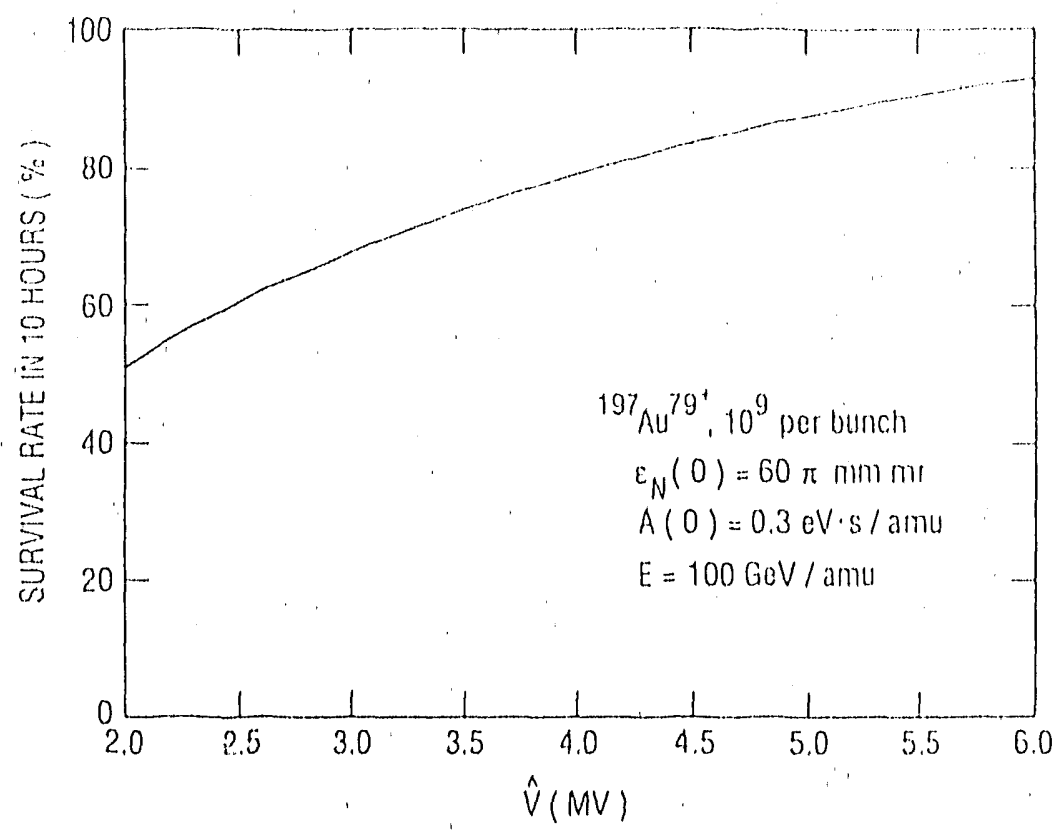

Figure 6: The final beam survival rate as a function of the peak rf voltage for the 10-hour operation using a constant rf voltage.

the initial conditions. During the operation the rms bunch length of varies from about $12 \mathrm{~cm}$ to $27 \mathrm{~cm}$.

Figs. 6 and 7 show the final beam survival rate and bunch lengths as functions of the constant peak voltage. A larger peak voltage provides a larger bucket area which allows a larger bunch. Both the loss rate and the growth rate of this larger bunch are smaller. Therefore, the total beam loss reduces with the increasing peak voltage.

\section{B. Tight bucket with initial emittance blow-up}

If a constant bunch length is required during the operation, the rf voltage has to be programmed to accommodate the beam growth due to intrabeam scattering. The "tight bucket" condition resulted from this programming implies that the ratio of the bucket area to the bunch area is a constant.

The criterion for the voltage programming adopted in our computer calculation is that .' 


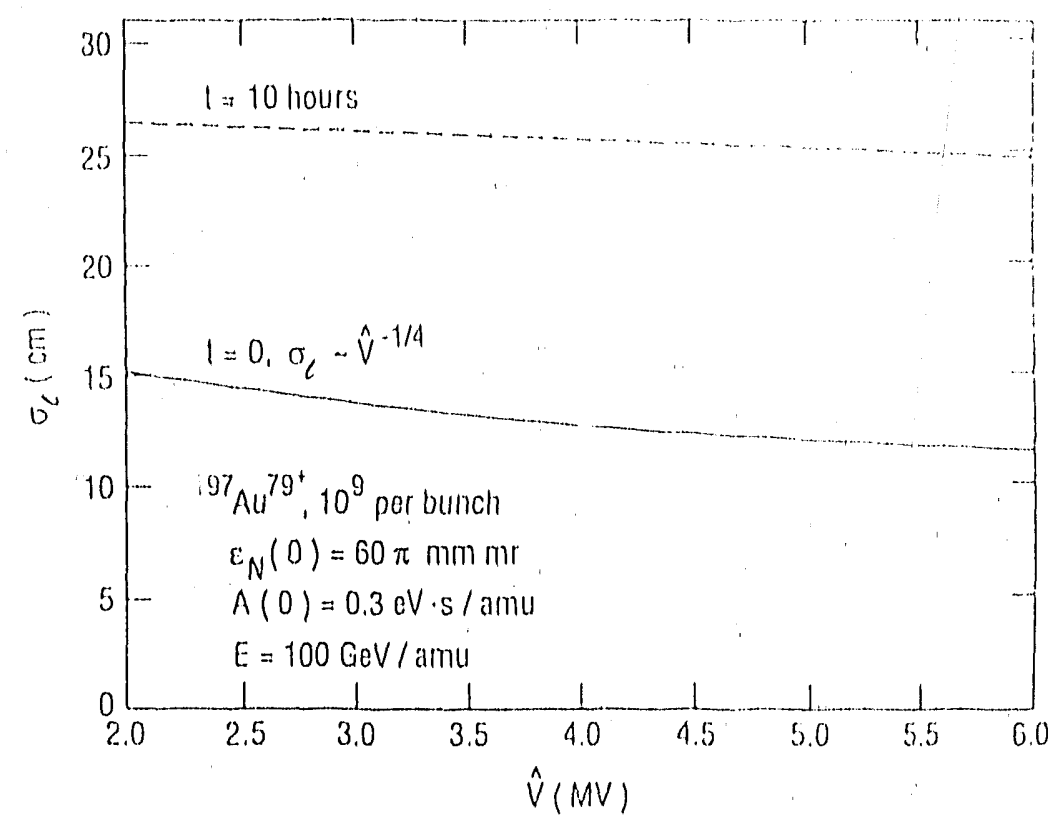

Figure 7: The initial and final bunch length as a function of the peak rf voltage for the 10-hour operation using a constant rf voltage.

the bucket area $A_{B}$ is about 3.5 times that of the average area $\langle J\rangle$. Correspondingly, the ratio of the maximum momentum spread $\Delta_{B}$ of the bucket to the rms momentum spread $\sigma_{\Delta p / p}$ of the bunch is $t$ about 2.5 . 'l'he programming of the peak voltage to satisfy this criterion is shown in fig. 8. During the early period of operation, the voltage increases drastically to accommodate the fast growth in bunch area. Since the bunch area is always comparable to the bucket area, beam loss is severe (fig. 9) during the entire period. The growth rate decreases with the increasing bunch size and the decreasing beam intensity. The required voltage reaches $4.5 \mathrm{MV}$ in 10 hours. The total bean loss is $50 \sim 60 \%$.

\section{Constant voltage without initial emittance blow-up}

The initial normalized transverse emittance without the blow-up is about $10 \pi \mathrm{mm}$. mrad. Due to this small value, the beam grows appreciably in both the transverse and the

The originally proposed tight-buckot critorion is $\Delta_{B} / \sigma_{\Delta p / p}=2$. However, this can not be realifed in the calculation when the beam loss at the boundary is taken into account. 


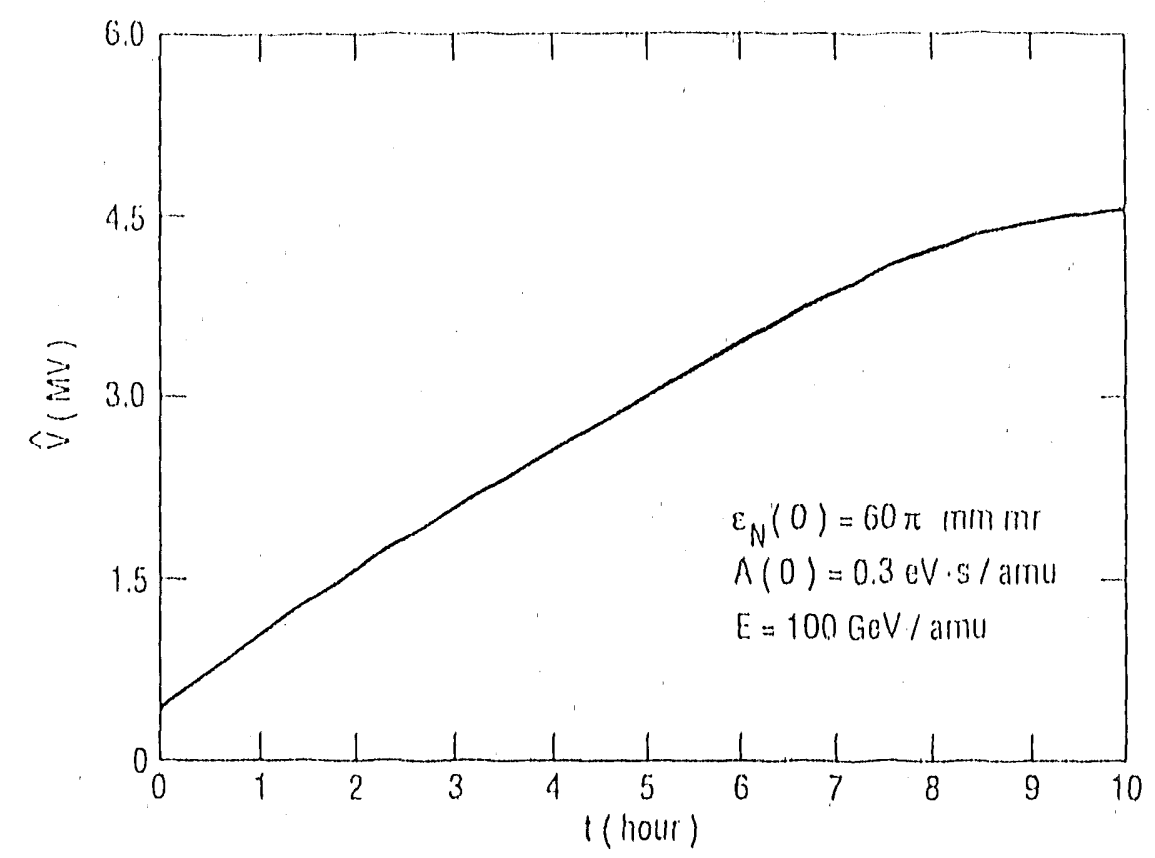

Figure 8: The programming of the peak rf voltage during the tight-bucket operation.

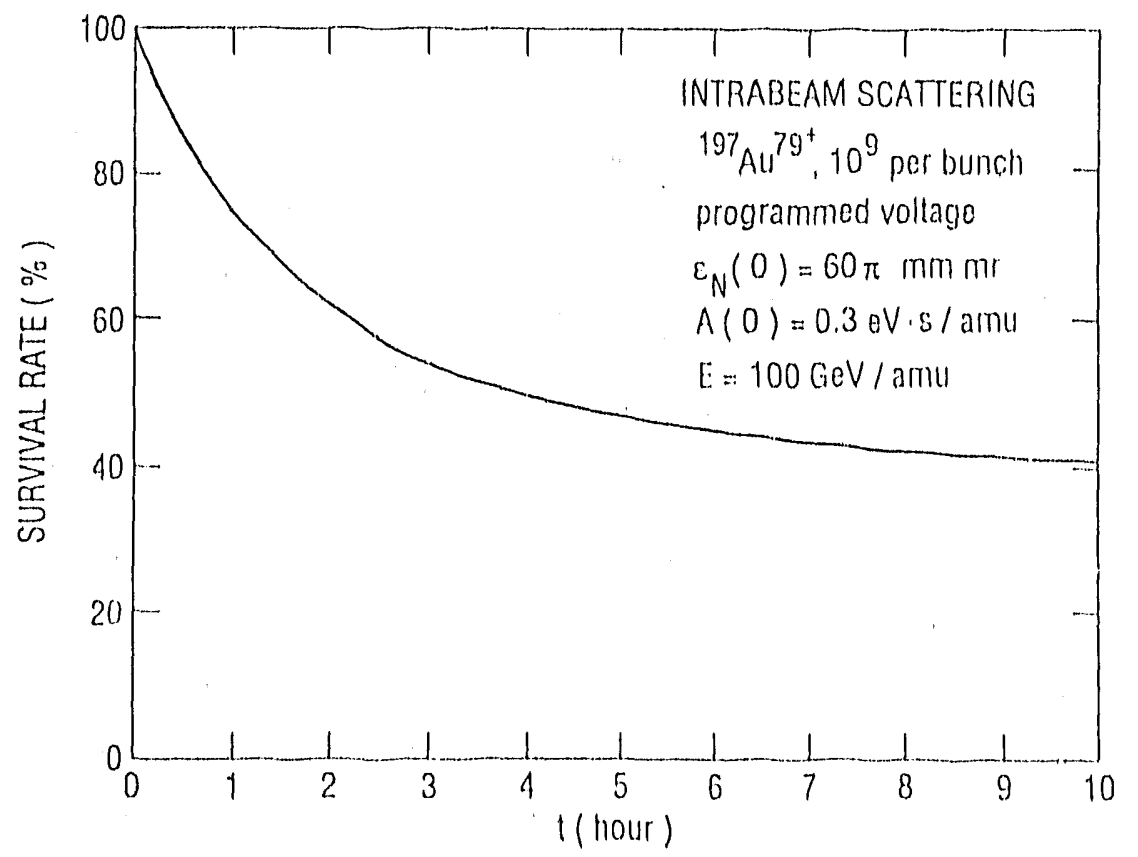

Figure 9: The beam survival rate during the tight-bucket operation. 
longitudinal directions. According to G.Parzen's calculation, ${ }^{14}$ the transverse enittance increases to about $34 \pi \mathrm{mm}$-mrth in 10 hours.

With a constant voltage of $4.5 \mathrm{MV}$, the beam loss in longitudinal direction is found to be $40 \%$. If a constant voltage of $11.5 \mathrm{MV}$ is achievable, the beam loss will be reduced to about $10 \%$.

\section{Discussion}

The calculation indicates that the most efficient and economical operational scenario consists of using a constant peak if voltage of 4.5MV during the entire 10-hour period

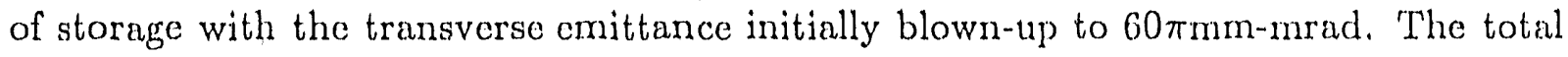
beam loss is about 20\%, while the luminosity ${ }^{\dagger}$ is reduced by about $40 \%$ in 10 hours mainly due to this beam loss. The averaged rms bunch length is about $23 \mathrm{~cm}$.

The situation will be significantly improved if measures like stochastic cooling are adopted.

\section{Acknowledgment}

We would like to express our sincerest thanks to Dr. G.Parzen for the valuable discussion and the very helpful information on transverse beam growth.

\section{REFERENCES}

1) A.Piwinski, Proc. CERN Accelerator School on General Acceleralor Physics, GifsurY'vette, Paris, 1984, p 405.

2) M.Martini, CERN PS/84-9 (AA) (1984).

3) J. Bjorken and S.Mtingwa, Particle Accelerators 13, 115 (1983).

In this case, the reduction in the average luminosity is about $15 \%$ compared with the calculation without considoring the boam loss. 
4) G.Parzen, Nucl. Instr. Meth. A256, 231 (1987).

5) Brookhaven National Laboratory, Conceptual Design of the RHIC, BNL-52195 (1989).

6) R.Cohen, L.Spitzer, and P.McRoutly, Phys. Rev. 80, 230 (1950).

7) S.Gasiorowicz, M.Neuman, and R.Riddell, Phys. Rev. 101, 922 (1956).

8) M.Rosenbluth, W.MacDonald, and D.Judd, Phys. Rev. 107, 1 (1957).

9) A.H. Sørensscn, Introduction to Intrabeam Scattering, Conference cuntribution, p.135.

10) M. Rhoudes-Brown and J.Claus, AD/AP-16, (1990).

11) J.Wei and A.G.Ruggiero, AD/RHIC-71 (1990).

12) H. Goldstein, Classical Mechanics (Addison-Wesley, New York, 1953).

13) I.S. Gradshteyn and I.M. Ryshik, Table of Integrals, Series, and Products (Academic, Now York, 1965).

14) G.Parzen, private communications. 

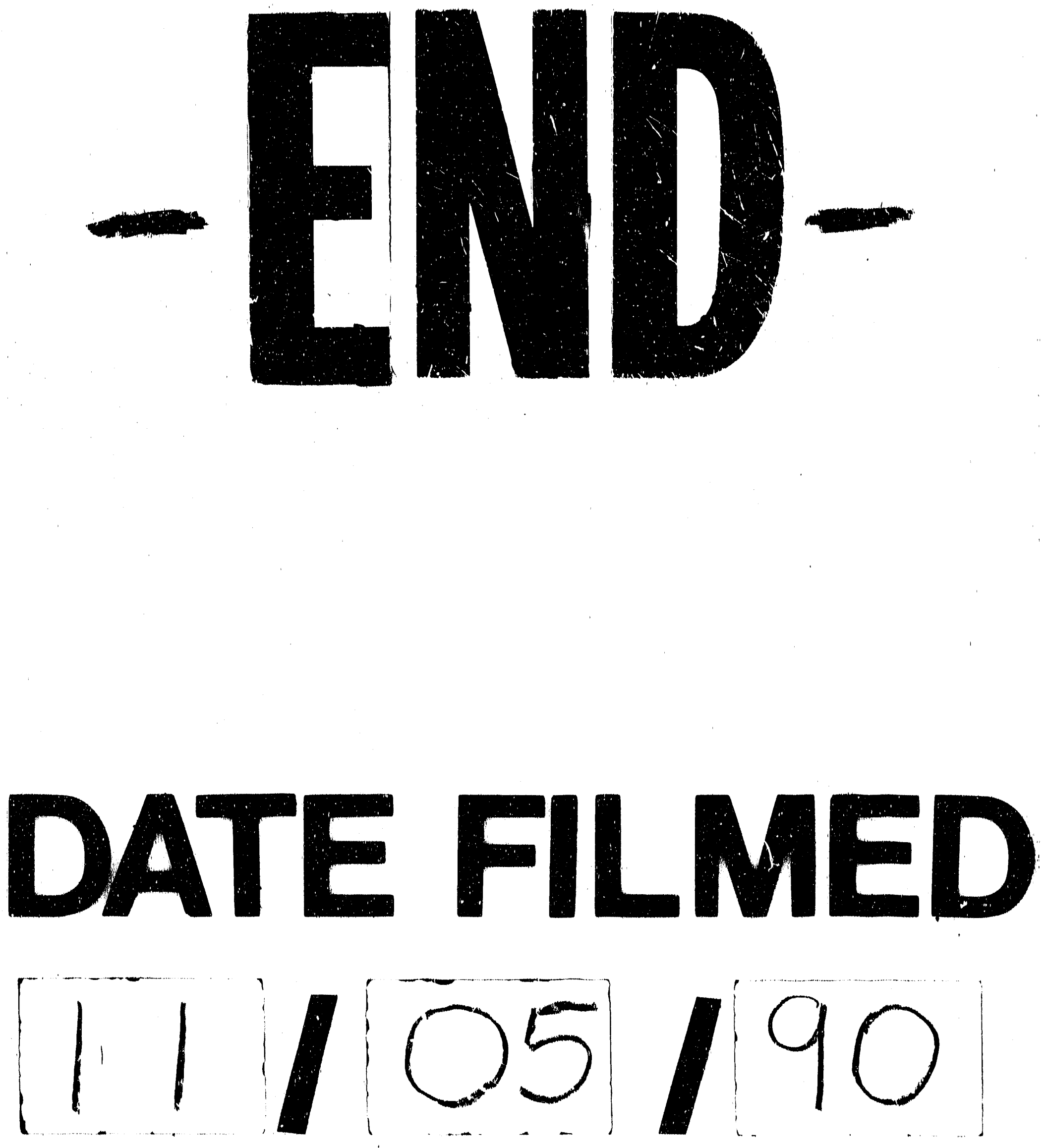
